\title{
Natural Partial Orders on Transformation Semigroups with Fixed Sets
}

\author{
Yanisa Chaiya, Preeyanuch Honyam, and Jintana Sanwong \\ Department of Mathematics, Chiang Mai University, Chiang Mai 50200, Thailand \\ Correspondence should be addressed to Jintana Sanwong; jintana.s@cmu.ac.th
}

Received 20 April 2016; Revised 1 July 2016; Accepted 5 July 2016

Academic Editor: Pentti Haukkanen

Copyright (C) 2016 Yanisa Chaiya et al. This is an open access article distributed under the Creative Commons Attribution License, which permits unrestricted use, distribution, and reproduction in any medium, provided the original work is properly cited.

Let $X$ be a nonempty set. For a fixed subset $Y$ of $X$, let $\operatorname{Fix}(X, Y)$ be the set of all self-maps on $X$ which fix all elements in $Y$. Then $\operatorname{Fix}(X, Y)$ is a regular monoid under the composition of maps. In this paper, we characterize the natural partial order on Fix $(X, Y)$ and this result extends the result due to Kowol and Mitsch. Further, we find elements which are compatible and describe minimal and maximal elements.

\section{Introduction}

For any semigroup $S$, the natural partial order on $E(S)$, the set of all idempotents on $S$, is defined by

$$
e \leq f \quad \text { iff } e=e f=f e .
$$

In 1980, Hartwig [1] and Nambooripad [2] proved that if $S$ is a regular semigroup, then the relation

$$
a \leq b \quad \text { iff } a=e b=b f \text { for some } e, f \in E(S)
$$

is a partial order on $S$ which extends the usual ordering of the set $E(S)$

Later in 1986, the natural partial order on a regular semigroup was further extended to any semigroup $S$ by Mitsch [3] as follows:

$$
\begin{array}{cl}
a \leq b & \text { iff } a=x b=b y, \\
x a=a & \text { for some } x, y \in S^{1} .
\end{array}
$$

Let $X$ be a set and $B(X)$ denote the semigroup of binary relations on the set $X$ under the composition of relations. A partial transformation semigroup is the collection of functions from a subset of $X$ into $X$ with composition which is denoted by $P(X)$. Let $T(X)$ be the set of all transformations from $X$ into itself and it is called the full transformation semigroup on $X$. Then $P(X)$ and $T(X)$ are subsemigroups of $B(X)$. It is well known that $P(X)$ and $T(X)$ are regular semigroups.

In 1986, Kowol and Mitsch [4] characterized the natural partial order on $T(X)$ in terms of images and kernels. They also proved that an element $\alpha \in T(X)$ is maximal with respect to the natural order if and only if $\alpha$ is surjective or injective; $\alpha$ is minimal if and only if $\alpha$ is a constant map. Moreover, they described lower and upper bounds for two transformations and gave necessary and sufficient conditions for their existence.

Later in 2006, Namnak and Preechasilp [5] studied two natural partial orders on $\mathrm{B}(X)$ and characterized when two elements of $B(X)$ are related under these orders. They also described the minimality, maximality, left compatibility, and right compatibility of elements with respect to each order.

Let $Y$ be a subset of $X$. Recently, Fernandes and Sanwong [6] defined

$$
P T(X, Y)=\{\alpha \in P(X): X \alpha \subseteq Y\},
$$

where $X \alpha$ denotes the image of $\alpha$. Moreover, they defined $I(X, Y)$ to be the set of all injective transformations in $P T(X, Y)$. Hence $P T(X, Y)$ and $I(X, Y)$ are subsemigroups of $P(X)$. 
In [7], Sangkhanan and Sanwong described natural partial order $\leq$ on $P T(X, Y)$ and $I(X, Y)$ in terms of domains, images, and kernels. They also compared $\leq$ with the subset order and characterized the meet and join of these two orders. Furthermore, they found elements of $P T(X, Y)$ and $I(X, Y)$ which are compatible and determined the minimal and maximal elements.

Let $Y$ be a fixed subset of $X$ and

$$
\operatorname{Fix}(X, Y)=\{\alpha \in T(X): y \alpha=y \forall y \in Y\} .
$$

In 2013, Honyam and Sanwong [8] proved that $\operatorname{Fix}(X, Y)$ is a regular semigroup and they also determined its Green's relations and ideals. Moreover, they proved that $\operatorname{Fix}(X, Y)$ is never isomorphic to $T(Z)$ for any set $Z$ when $\emptyset \neq Y \subsetneq X$, and every semigroup $S$ is isomorphic to a subsemigroup of $\operatorname{Fix}\left(X^{\prime}, Y^{\prime}\right)$ for some appropriate sets $X^{\prime}$ and $Y^{\prime}$ with $Y^{\prime} \subseteq$ $X^{\prime}$. Note that this also follows trivially from the fact that $T(X)$ embeds in $\operatorname{Fix}(X \cup Z, Z)$ for any set $Z$ with $X \cap Z=$ $\emptyset$. Recently, the authors in [9] proved that there are only three types of maximal subsemigroups of $\operatorname{Fix}(X, Y)$ and these maximal subsemigroups coincide with the maximal regular subsemigroups when $X \backslash Y$ is a finite set with $|X \backslash Y| \geq 2$. They also gave necessary and sufficient conditions for $\operatorname{Fix}(X, Y)$ to be factorizable, unit-regular, and directly finite.

In this paper, we characterize the natural partial order on $\operatorname{Fix}(X, Y)$ and find elements which are compatible under this order in Section 3. In Section 4, we describe the minimal elements, the maximal elements, and the covering elements. Moreover, we find the number of upper covers of minimal elements and the number of lower covers of maximal elements.

\section{Preliminaries and Notations}

In [8], the authors proved that $\operatorname{Fix}(X, Y)$ is a regular subsemigroup of $T(X)$. Note that $\operatorname{Fix}(X, Y)$ contains $1_{X}$, the identity map on $X$. If $Y=\emptyset$, then $\operatorname{Fix}(X, Y)=T(X)$; and if $|X|=1$ or $X=Y$, then $\operatorname{Fix}(X, Y)$ consists of one element, $1_{X}$. So, throughout this paper we will consider the case $Y \subsetneq X$ and $|X|>1$.

For any $\alpha \in T(X)$, the symbol $\pi_{\alpha}$ denotes the partition of $X$ induced by the map $\alpha$, namely,

$$
\pi_{\alpha}=\left\{x \alpha^{-1}: x \in X \alpha\right\} .
$$

For $\alpha, \beta \in T(X), \mathscr{A} \subseteq \pi_{\alpha}$, and $\mathscr{B} \subseteq \pi_{\beta}$, we say that $\mathscr{A}$ refines $\mathscr{B}$ if for each $A \in \mathscr{A}$ there exists $B \in \mathscr{B}$ such that $A \subseteq B$.

Throughout this paper, unless otherwise stated, let $Y=$ $\left\{y_{i}: i \in I\right\}$.

For each $\alpha \in \operatorname{Fix}(X, Y)$, we have $y_{i} \alpha=y_{i}$ for all $i \in I$. So $Y=Y \alpha \subseteq X \alpha$. If $\alpha \in \operatorname{Fix}(X, Y)$, then we write

$$
\alpha=\left(\begin{array}{ll}
A_{i} & B_{j} \\
y_{i} & b_{j}
\end{array}\right)
$$

and take as understood that the subscripts $i$ and $j$ belong to the index sets $I$ and $J$, respectively, such that $X \alpha=\left\{y_{i}: i \in\right.$ $I\} \cup\left\{b_{j}: j \in J\right\}, y_{i} \alpha^{-1}=A_{i}$, and $b_{j} \alpha^{-1}=B_{j}$. Thus $A_{i} \cap Y=\left\{y_{i}\right\}$ for all $i \in I, B_{j} \subseteq X \backslash Y$ for all $j \in J$ and $\left\{b_{j}: j \in J\right\} \subseteq X \backslash Y$. Here $J$ can be an empty set.

An idempotent $e$ in a semigroup $S$ is said to be minimal if $e$ has the property $f \in E(S)$ and $f \leq e$ implies $f=e$.

In [8] the authors showed that

$$
\begin{aligned}
E_{m} & =\left\{\left(\begin{array}{c}
A_{i} \\
y_{i}
\end{array}\right):\left\{A_{i}: i \in I\right\} \text { is a partition of } X \text { with } y_{i}\right. \\
& \left.\in A_{i}\right\}
\end{aligned}
$$

is the set of all minimal idempotents in $\operatorname{Fix}(X, Y)$ and it is an ideal of $\operatorname{Fix}(X, Y)$. We note that $E_{m}$ is simply the set $\{\alpha \in$ $\operatorname{Fix}(X, Y): X \alpha=Y\}$ and $\alpha$ is an idempotent in $\operatorname{Fix}(X, Y)$ if and only if $x \alpha=x$ for all $x \in X \alpha \backslash Y$.

\section{Natural Partial Order on $\operatorname{Fix}(X, Y)$}

Kowol and Mitsch [4] gave a characterization of the natural partial order on $T(X)$. Later in 1994, Higgins [10] showed that if $T$ is a regular subsemigroup of a semigroup $S$, then the natural partial order on $T$ is the restriction to $T$ of the natural partial order on $S$. Here we describe the natural partial order on $\operatorname{Fix}(X, Y)$ which is a regular subsemigroup of $T(X)$ without making use of Higgins' result and when we take $Y=$ $\emptyset$, we recapture the result above by Kowol and Mitsch.

We note that if $\alpha, \beta \in \operatorname{Fix}(X, Y)$ and $\alpha=\beta \gamma$ for some $\gamma \in \operatorname{Fix}(X, Y)$, then $\pi_{\beta}$ refines $\pi_{\alpha}$.

Since $\operatorname{Fix}(X, Y)$ is regular, we use $(*)$ to study the natural partial order on this semigroup.

Theorem 1. Let $\alpha, \beta \in \operatorname{Fix}(X, Y)$. Then $\alpha \leq \beta$ if and only if the following statements hold:

(1) $X \alpha \subseteq X \beta$;

(2) $\pi_{\beta}$ refines $\pi_{\alpha}$;

(3) if $x \beta \in X \alpha$, then $x \beta=x \alpha$.

Proof. Suppose that $\alpha \leq \beta$. Then, by $(*)$, we have

$$
\alpha=\lambda \beta=\beta \gamma
$$

for some $\lambda, \gamma \in E(\operatorname{Fix}(X, Y))$. Thus $X \alpha=(X \lambda) \beta \subseteq X \beta$. Since $\alpha=\beta \gamma$, we get that $\pi_{\beta}$ refines $\pi_{\alpha}$. Now, let $x \beta \in X \alpha$. Then $x \beta=x^{\prime} \alpha$ for some $x^{\prime} \in X$ and thus $x \beta=x^{\prime} \alpha=x^{\prime} \beta \gamma=$ $\left(x^{\prime} \beta\right) \gamma$. Hence $x \beta \in X \gamma$ and then $x \alpha=x \beta \gamma=x \beta$ since $\gamma$ is an idempotent.

Conversely, assume that conditions (1)-(3) hold. By condition (1), we can write

$$
\begin{aligned}
& \alpha=\left(\begin{array}{ll}
A_{i} & B_{j} \\
y_{i} & b_{j}
\end{array}\right), \\
& \beta=\left(\begin{array}{lll}
A_{i}^{\prime} & C_{j} & C_{k} \\
y_{i} & b_{j} & b_{k}
\end{array}\right),
\end{aligned}
$$

where $y_{i} \in A_{i} \cap A_{i}^{\prime}, b_{j}, b_{k} \in X \backslash Y$, and $B_{j}, C_{j}, C_{k} \subseteq X \backslash Y$. Since $y_{i} \in A_{i} \cap A_{i}^{\prime}$ and $\pi_{\beta}$ refines $\pi_{\alpha}$, we obtain $A_{i}^{\prime} \subseteq A_{i}$ for all $i \in I$. 
If $J=\emptyset$, then define $\lambda=\alpha$ and thus $\alpha=\lambda \beta$. If $J \neq \emptyset$, then, for each $j \in J$, let $c_{j} \in C_{j}$. So $c_{j} \beta=b_{j} \in X \alpha$. By condition (3), $c_{j} \alpha=c_{j} \beta=b_{j}$; that is, $c_{j} \in B_{j}$ and hence $C_{j} \subseteq B_{j}$. Define

$$
\lambda=\left(\begin{array}{cc}
A_{i} & B_{j} \\
y_{i} & c_{j}
\end{array}\right) .
$$

We get $\lambda \in E(\operatorname{Fix}(X, Y))$ and $\alpha=\lambda \beta$.

If $K=\emptyset$, then $\alpha=\beta 1_{X}$. If $K \neq \emptyset$, then, for each $k \in K$, we choose $c_{k} \in C_{k}$.

Case 1. Consider $X \beta=X$. Then $X \backslash X \alpha=\left\{b_{k}: k \in K\right\}$. We define $\gamma \in \operatorname{Fix}(X, Y)$ by

$$
x \gamma= \begin{cases}x, & x \in X \alpha \\ c_{k} \alpha, & x=b_{k} \in X \backslash X \alpha .\end{cases}
$$

To prove that $\alpha=\beta \gamma$, let $x \in X$. If $x \in A_{i}^{\prime}$ for some $i$ or $x \in C_{j}$ for some $j$, then it is clear that $x \alpha=x \beta \gamma$. Now, if $x \in C_{k}$ for some $k$, then $x \beta=c_{k} \beta$ and thus $x \alpha=c_{k} \alpha$ since $\pi_{\beta}$ refines $\pi_{\alpha}$. So $(x \beta) \gamma=b_{k} \gamma=c_{k} \alpha=x \alpha$. Hence $\alpha=\beta \gamma$. It remains to show that $\gamma$ is an idempotent. Let $x \gamma \in X \gamma \backslash X \alpha$. Then $x \gamma=c_{k} \alpha$ for some $k$. Thus $(x \gamma) \gamma=\left(c_{k} \alpha\right) \gamma=c_{k} \alpha=x \gamma$ since $c_{k} \alpha \in X \alpha$.

Case 2. Consider $X \beta \subsetneq X$. We choose $c_{0} \in X \backslash X \beta$ and define $\gamma^{\prime} \in \operatorname{Fix}(X, Y)$ by

$$
x \gamma^{\prime}= \begin{cases}x, & x \in X \alpha, \\ c_{k} \alpha, & x=b_{k} \in X \beta \backslash X \alpha, \\ c_{0}, & x \in X \backslash X \beta .\end{cases}
$$

By the same prove as given in Case 1, we get $\alpha=\beta \gamma^{\prime}$ and $\left(x \gamma^{\prime}\right) \gamma^{\prime}=x \gamma^{\prime}$ for all $x \gamma^{\prime} \in X \beta$. If $x \gamma^{\prime}=c_{0}$, then $\left(x \gamma^{\prime}\right) \gamma^{\prime}=$ $c_{0} \gamma^{\prime}=c_{0}=x \gamma^{\prime}$. So $\gamma^{\prime}$ is an idempotent. Therefore, $\alpha \leq \beta$ by $(*)$.

Remark 2. If $Y=\emptyset$, then $\operatorname{Fix}(X, Y)=T(X)$, and we have the characterization of $\leq$ on $T(X)$ which first appeared in $[4$, Proposition 2.3].

As a direct consequence of Theorem 1, we get the following corollary.

Corollary 3. Let $\alpha, \beta \in \operatorname{Fix}(X, Y)$ with $\alpha \leq \beta$. If $X \alpha \backslash Y=$ $X \beta \backslash Y$, then $\alpha=\beta$.

Let $S$ be a semigroup. An element $a \in S$ is said to be left (right) compatible with respect to the partial order $\leq$ if $a b \leq$ $a c(b a \leq c a)$ whenever $b \leq c$.

The following results describe all the left compatible and right compatible elements in $\operatorname{Fix}(X, Y)$ when $\emptyset \neq Y \subsetneq X$. We also write $\alpha<\beta$ instead of $\alpha \leq \beta$ and $\alpha \neq \beta$ for $\alpha, \beta \in$ $\operatorname{Fix}(X, Y)$.

Theorem 4. Assume that $\emptyset \neq Y \subsetneq X$ and let $\lambda \in$ $\operatorname{Fix}(X, Y)$. Then $\lambda$ is left compatible if and only if $\lambda$ is a minimal idempotent or $\lambda$ is surjective.
Proof. Suppose that $\lambda$ is left compatible. Assume by contrary that $\lambda$ is not a minimal idempotent and $\lambda$ is not surjective. So there are $a \in X \lambda \backslash Y$ and $b \in X \backslash X \lambda$. Define

$$
\begin{aligned}
& \alpha=\left(\begin{array}{cc}
y_{i} & X \backslash Y \\
y_{i} & a
\end{array}\right), \\
& \beta=\left(\begin{array}{ccc}
y_{i} & b & X \backslash(Y \cup\{b\}) \\
y_{i} & a & b
\end{array}\right) .
\end{aligned}
$$

Then $\alpha, \beta \in \operatorname{Fix}(X, Y)$ with $\alpha<\beta$ and thus $\lambda \alpha \leq \lambda \beta$ since $\lambda$ is left compatible. However, $X \lambda \alpha \nsubseteq X \lambda \beta$ since $a \in X \lambda \alpha$ but $a \notin X \lambda \beta$, a contradiction.

Conversely, let $\alpha \leq \beta$. If $\lambda$ is a minimal idempotent, then $\lambda \alpha=\lambda=\lambda \beta$. Now, assume that $\lambda$ is surjective. So $X \lambda \alpha=$ $X \alpha \subseteq X \beta=X \lambda \beta$. Let $A \in \pi_{\lambda \beta}$. So $A=x(\lambda \beta)^{-1}=\left(x \beta^{-1}\right) \lambda^{-1}$ for some $x \in X \lambda \beta$. Since $\alpha \leq \beta$, we have that $\pi_{\beta}$ refines $\pi_{\alpha}$ and hence $x \beta^{-1} \subseteq x^{\prime} \alpha^{-1}$ for some $x^{\prime} \in X \alpha$. Since $x^{\prime} \in X \alpha$, we get $x^{\prime}=u \alpha$ for some $u \in X$ and $u=v \lambda$ for some $v \in X$ because $\lambda$ is surjective. Hence $v \lambda \alpha=u \alpha=x^{\prime}$; that is, $x^{\prime} \in X \lambda \alpha$. Further, $A=\left(x \beta^{-1}\right) \lambda^{-1} \subseteq\left(x^{\prime} \alpha^{-1}\right) \lambda^{-1}=x^{\prime}(\lambda \alpha)^{-1} \in \pi_{\lambda \alpha}$, thus $\pi_{\lambda \beta}$ refines $\pi_{\lambda \alpha}$. Let $a \lambda \beta \in X \lambda \alpha$. So $(a \lambda) \beta \in X \alpha$ and then $a \lambda \beta=a \lambda \alpha$. By Theorem 1 , we have $\lambda \alpha \leq \lambda \beta$ which implies that $\lambda$ is left compatible.

Theorem 5. The following statements hold.

(1) If $|Y|=1$, then $\lambda \in \operatorname{Fix}(X, Y)$ is right compatible if and only if $\lambda$ is a minimal idempotent or $\lambda$ is injective.

(2) If $|Y| \geq 2$, then $\lambda \in \operatorname{Fix}(X, Y)$ is right compatible if and only if $\lambda$ is injective.

Proof. (1) Assume that $Y=\{y\}$ and $\lambda$ is right compatible. Suppose in the contrary that $\lambda$ is not a minimal idempotent and $\lambda$ is not injective. So we can write

$$
\lambda=\left(\begin{array}{ll}
A & B_{j} \\
y & b_{j}
\end{array}\right),
$$

where $y \in A$ and $J \neq \emptyset$. Since $\lambda$ is not injective, two cases arise.

Case 1. Consider $|A| \geq 2$. Choose $a \in A \backslash\{y\}$ and $c \in B_{j_{0}}$ for some $j_{0} \in J$. Let $X \backslash\{a, c\}=\left\{x_{k}: k \in K\right\}$ and define $\alpha \in \operatorname{Fix}(X, Y)$ by

$$
\alpha=\left(\begin{array}{cc}
\{a, c\} & x_{k} \\
c & x_{k}
\end{array}\right)
$$

we get $\alpha<1_{X}$. Moreover, we have $a\left(1_{X} \lambda\right)=a \lambda=y=$ $y\left(1_{X} \lambda\right)$, hence there is $B \in \pi_{1_{X} \lambda}$ such that $\{a, y\} \subseteq B$. However, $\{a, y\} \nsubseteq C$ for all $C \in \pi_{\alpha \lambda}$ since $a(\alpha \lambda)=c \lambda=$ $b_{j_{0}} \neq y=y(\alpha \lambda)$. This means that $\pi_{1_{X} \lambda}$ does not refine $\pi_{\alpha \lambda}$. By Theorem 1, we get $\alpha \lambda \npreceq 1_{X} \lambda$, a contradiction. 
Case 2. Consider $\left|B_{j_{0}}\right| \geq 2$ for some $j_{0} \in J$. Choose $a, b \in B_{j_{0}}$ such that $a \neq b$. Let $X \backslash\{a, b, y\}=\left\{x_{k}: k \in K\right\}$. Define $\alpha, \beta \in \operatorname{Fix}(X, Y)$ by

$$
\begin{aligned}
& \alpha=\left(\begin{array}{ccc}
\{y, a\} & b & x_{k} \\
y & a & x_{k}
\end{array}\right), \\
& \beta=\left(\begin{array}{llll}
a & b & y & x_{k} \\
b & a & y & x_{k}
\end{array}\right),
\end{aligned}
$$

we get $\alpha<\beta$. Since $a(\beta \lambda)=b \lambda=a \lambda=b(\beta \lambda)$, there is $B \in \pi_{\beta \lambda}$ such that $\{a, b\} \subseteq B$. However, $\{a, b\} \nsubseteq C$ for all $C \in \pi_{\alpha \lambda}$ since $a(\alpha \lambda)=y \lambda=y \neq b_{j_{0}}=a \lambda=b(\alpha \lambda)$. So $\pi_{\beta \lambda}$ does not refine $\pi_{\alpha \lambda}$. By Theorem 1 , we get $\alpha \lambda \nless \_\beta \lambda$, a contradiction.

Conversely, let $\alpha, \beta \in \operatorname{Fix}(X, Y)$ be such that $\alpha \leq \beta$. If $\lambda$ is a minimal idempotent, then $\lambda=\left(\begin{array}{l}X \\ y\end{array}\right)$ and $\alpha \lambda=\lambda=\beta \lambda$; that is, $\lambda$ is right compatible. Now, assume that $\lambda$ is injective. Since $X \alpha \subseteq X \beta$, we get $X \alpha \lambda \subseteq X \beta \lambda$. Let $A \in \pi_{\beta \lambda}$. So $A=x(\beta \lambda)^{-1}=\left(x \lambda^{-1}\right) \beta^{-1}$ for some $x \in X \beta \lambda$ and hence $\left(x \lambda^{-1}\right) \beta^{-1} \subseteq x^{\prime} \alpha^{-1}$ for some $x^{\prime} \in X \alpha$. So $x^{\prime}=u \alpha$ for some $u \in X$. Since $\lambda$ is injective, $\left\{x^{\prime}\right\}=v \lambda^{-1}$ for some $v \in X \lambda$ and $u \alpha \lambda=x^{\prime} \lambda=v$; that is, $v \in X \alpha \lambda$. Thus $x^{\prime} \alpha^{-1}=\left(v \lambda^{-1}\right) \alpha^{-1}=v(\alpha \lambda)^{-1} \in \pi_{\alpha \lambda}$ which implies that $\pi_{\beta \lambda}$ refines $\pi_{\alpha \lambda}$. Let $a \beta \lambda \in X \alpha \lambda$. So $a \beta \lambda=b \alpha \lambda$ for some $b \in X$. Since $\lambda$ is injective, $a \beta=b \alpha$ and then $a \beta \in X \alpha$. Thus $a \beta=a \alpha$ since $\alpha \leq \beta$ and that $a \beta \lambda=a \alpha \lambda$. Therefore, $\alpha \lambda \leq \beta \lambda$, and we conclude that $\lambda$ is right compatible.

(2) Suppose that $\lambda$ is right compatible and $\lambda$ is not injective. Write

$$
\lambda=\left(\begin{array}{cc}
A_{i} & B_{j} \\
y_{i} & b_{j}
\end{array}\right),
$$

where $y_{i} \in A_{i}$ and $|I| \geq 2$. Since $\lambda$ is not injective, two cases arise.

Case 1. $\left|A_{i_{0}}\right| \geq 2$ for some $i_{0} \in I$. Choose $a \in A_{i_{0}} \backslash\left\{y_{i_{0}}\right\}$ and $y_{i_{1}} \in Y \backslash\left\{y_{i_{0}}\right\}$. Let $X \backslash\left\{y_{i_{1}}, a\right\}=\left\{x_{k}: k \in K\right\}$ and define

$$
\alpha=\left(\begin{array}{cc}
\left\{y_{i_{1}}, a\right\} & x_{k} \\
y_{i_{1}} & x_{k}
\end{array}\right) .
$$

Then $\alpha<1_{X}$ and hence $\alpha \lambda \leq 1_{X} \lambda$. We can see that $\left\{y_{i_{0}}, a\right\} \subseteq$ $A_{i_{0}} \in \pi_{\lambda}=\pi_{1_{X} \lambda}$, but $\left\{y_{i_{0}}, a\right\} \nsubseteq B$ for all $B \in \pi_{\alpha \lambda}$ since $a \alpha \lambda=$ $y_{i_{1}} \lambda=y_{i_{1}} \neq y_{i_{0}}=y_{i_{0}} \alpha \lambda$. This means that $\pi_{1_{X} \lambda}$ does not refine $\pi_{\alpha \lambda}$, a contradiction.

Case 2. $\left|B_{j_{0}}\right| \geq 2$ for some $j_{0} \in J$. This is virtually identical to Case 2 of (1) above.

\section{Minimal and Maximal Elements}

Let $S$ be a semigroup together with the partial order $\leq$. $S$ is said to be directed downward if every pair of elements has a lower bound. In other words, for any $a$ and $b$ in $S$, there exists $c$ in $S$ with $c \leq a$ and $c \leq b$. A directed upward semigroup is defined dually.
If $Y=\emptyset$, then $\operatorname{Fix}(X, Y)=T(X)$ and it has neither minimum nor maximum elements under the natural order (see [4]). So, in Lemmas 6 and 7 we assume that $\emptyset \neq Y \subsetneq X$.

Lemma 6. Assume that $\emptyset \neq Y \subsetneq X$. Then the following statements are equivalent.

(1) $\operatorname{Fix}(X, Y)$ has a minimum element.

(2) $\operatorname{Fix}(X, Y)$ is directed downward.

(3) $|Y|=1$.

Proof. $(1) \Rightarrow(2)$ This is clear.

$(2) \Rightarrow(3)$ Assume that $\operatorname{Fix}(X, Y)$ is directed downward. Let $y_{i_{1}}, y_{i_{2}} \in Y$ and $J=I \backslash\left\{i_{1}, i_{2}\right\}$. Consider

$$
\begin{aligned}
& \alpha=\left(\begin{array}{ccc}
y_{j} & y_{i_{2}} & (X \backslash Y) \cup\left\{y_{i_{1}}\right\} \\
y_{j} & y_{i_{2}} & y_{i_{1}}
\end{array}\right), \\
& \beta=\left(\begin{array}{ccc}
y_{j} & y_{i_{1}} & (X \backslash Y) \cup\left\{y_{i_{2}}\right\} \\
y_{j} & y_{i_{1}} & y_{i_{2}}
\end{array}\right) .
\end{aligned}
$$

We have $\alpha, \beta \in \operatorname{Fix}(X, Y)$ and there is $\gamma \in \operatorname{Fix}(X, Y)$ such that $\gamma \leq \alpha$ and $\gamma \leq \beta$. By Theorem $1, \pi_{\alpha}$ refines $\pi_{\gamma}$ and $\pi_{\beta}$ refines $\pi_{\gamma}$. Then there is $A \in \pi_{\gamma}$ such that $(X \backslash Y) \cup\left\{y_{i_{1}}\right\} \subseteq A$ and $(X \backslash Y) \cup\left\{y_{i_{2}}\right\} \subseteq A$. Thus $y_{i_{1}}, y_{i_{2}} \in A$ and hence $y_{i_{1}}=y_{i_{2}}$. Since $y_{i_{1}}, y_{i_{2}}$ are arbitrary elements in $Y$, we obtain that $|Y|=1$.

$(3) \Rightarrow(1)$ Assume that $Y=\{y\}$. It is easy to see that $\theta=$ $\left(\begin{array}{l}X \\ y\end{array}\right)$ is the minimum element in $\operatorname{Fix}(X, Y)$.

Lemma 7. Assume that $\emptyset \neq Y \subsetneq X$. Then the following statements are equivalent.

(1) $\operatorname{Fix}(X, Y)$ has a maximum element.

(2) $\operatorname{Fix}(X, Y)$ is directed upward.

(3) $|X \backslash Y|=1$.

Proof. $(1) \Rightarrow(2)$ This is clear.

$(2) \Rightarrow(3)$ Assume that $\operatorname{Fix}(X, Y)$ is directed upward. Let $a, b \in X \backslash Y$ and $X \backslash\{a, b\}=\left\{x_{k}: k \in K\right\}$. Define

$$
\alpha=\left(\begin{array}{lll}
a & b & x_{k} \\
b & a & x_{k}
\end{array}\right) \in \operatorname{Fix}(X, Y) .
$$

Then there is $\gamma \in \operatorname{Fix}(X, Y)$ such that $\alpha \leq \gamma$ and $1_{X} \leq \gamma$. Since $\alpha$ and $1_{X}$ are bijective, $\gamma$ is also bijective and thus $b \gamma \in$ $(X \alpha \backslash Y) \cap\left(X 1_{X} \backslash Y\right)$. So $a=b \alpha=b \gamma=b 1_{X}=b$. Since $a, b$ are arbitrary elements in $X \backslash Y$, we get $|X \backslash Y|=1$.

(3) $\Rightarrow(1)$ Assume that $|X \backslash Y|=1$. It is easy to see that $1_{X}$ is the maximum element in $\operatorname{Fix}(X, Y)$.

We now describe minimal and maximal elements in $\operatorname{Fix}(X, Y)$ when $\emptyset \neq Y \subsetneq X$. If $|Y|=1$, then $\operatorname{Fix}(X, Y)$ has a minimum element by Lemma 6 and it is minimal. In the same way, if $|X \backslash Y|=1$, then $\operatorname{Fix}(X, Y)$ has a maximum element by Lemma 7 and it is maximal.

Theorem 8. Assume that $\emptyset \neq Y \subsetneq X$ and let $\alpha \in \operatorname{Fix}(X, Y)$. Then $\alpha$ is minimal if and only if $\alpha$ is a minimal idempotent. 
Proof. Assume that $\alpha$ is minimal but $\alpha$ is not a minimal idempotent. So we can write

$$
\alpha=\left(\begin{array}{ll}
A_{i} & B_{j} \\
y_{i} & b_{j}
\end{array}\right),
$$

where $J \neq \emptyset$. Choose $i_{0} \in I$ and $j_{0} \in J$. Let $I^{\prime}=I \backslash\left\{i_{0}\right\}$, $J^{\prime}=J \backslash\left\{j_{0}\right\}$ and define $\beta \in \operatorname{Fix}(X, Y)$ by

$$
\beta=\left(\begin{array}{ccc}
A_{i_{0}} \cup B_{j_{0}} & A_{i^{\prime}} & B_{j^{\prime}} \\
y_{i_{0}} & y_{i^{\prime}} & b_{j^{\prime}}
\end{array}\right) .
$$

Hence $\beta<\alpha$, which contradicts the minimality of $\alpha$.

Conversely, assume that $\alpha$ is a minimal idempotent and $\beta \leq \alpha$. Since $Y \subseteq X \beta \subseteq X \alpha=Y$, we get $X \beta=X \alpha$ and hence $X \beta \backslash Y \subseteq X \alpha \backslash Y$. By Corollary 3, we obtain $\beta=\alpha$.

Theorem 9. Assume that $\emptyset \neq Y \subsetneq X$ and let $\alpha \in \operatorname{Fix}(X, Y)$. Then $\alpha$ is maximal if and only if $\alpha$ is injective or $\alpha$ is surjective.

Proof. Let $\alpha$ be maximal. Assume that $\alpha$ is not injective and surjective. So there are $a, b, c \in X$ such that $a \alpha=b \alpha$ with $a \neq b$ and $c \in X \backslash X \alpha$. Write

$$
\alpha=\left(\begin{array}{ll}
A_{i} & B_{j} \\
y_{i} & b_{j}
\end{array}\right) .
$$

Case 1. $a, b \in A_{i_{0}}$ for some $i_{0} \in I$. We may assume that $a \neq y_{i_{0}}$. Let $I^{\prime}=I \backslash\left\{i_{0}\right\}$ and define

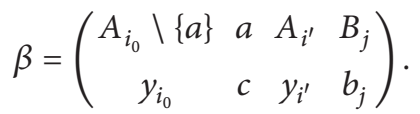

Then $\beta \in \operatorname{Fix}(X, Y)$ and $\alpha<\beta$ which contradicts the maximality of $\alpha$.

Case 2. $a, b \in B_{j_{0}}$ for some $j_{0} \in J$. Then we let $J^{\prime}=J \backslash\left\{j_{0}\right\}$ and define

$$
\gamma=\left(\begin{array}{ccccc}
A_{i} & B_{j_{0}} \backslash\{a\} & a & B_{j^{\prime}} \\
y_{i} & b_{j_{0}} & c & b_{j^{\prime}}
\end{array}\right) .
$$

Then $\gamma \in \operatorname{Fix}(X, Y)$ and $\alpha<\gamma$ which contradicts the maximality of $\alpha$.

Conversely, assume that $\alpha$ is injective or $\alpha$ is surjective and $\alpha \leq \beta$ for some $\beta \in \operatorname{Fix}(X, Y)$. Then $X \alpha \subseteq X \beta$ and $X \alpha \backslash$ $Y \subseteq X \beta \backslash Y$. Consider the case where $\alpha$ is injective, by letting $z \in X \beta \backslash Y$. Then $z=x \beta$ for some $x \in X \backslash Y$ and $x \alpha \in$ $X \alpha \backslash Y \subseteq X \beta \backslash Y$; that is, $x \alpha=x^{\prime} \beta$ for some $x^{\prime} \in X \backslash Y$. So $x^{\prime} \beta \in X \alpha$ and $x^{\prime} \beta=x^{\prime} \alpha$ by Theorem 1. Since $\alpha$ is injective, we get $x=x^{\prime}$ and thus $z=x \beta=x^{\prime} \beta=x \alpha \in X \alpha \backslash Y$, whence $X \beta \backslash Y \subseteq X \alpha \backslash Y$. Hence, in this case, $X \alpha \backslash Y=X \beta \backslash Y$ and by Corollary 3 we obtain $\alpha=\beta$. In the case $\alpha$ is surjective, we get $X \backslash Y=X \alpha \backslash Y \subseteq X \beta \backslash Y \subseteq X \backslash Y$; that is, $X \alpha \backslash Y=X \beta \backslash Y$. Again by Corollary 3, we have that $\alpha=\beta$. Therefore, $\alpha$ is maximal.

Figure 1 shows the diagram of $\operatorname{Fix}(X, Y)$ when $X=$ $\{1,2,3,4\}$ and $Y=\{1,2\}$. The notation $(a b c d)$ for $\alpha \in$ $\operatorname{Fix}(X, Y)$ means that $1 \alpha=a, 2 \alpha=b, 3 \alpha=c$, and $4 \alpha=d$.

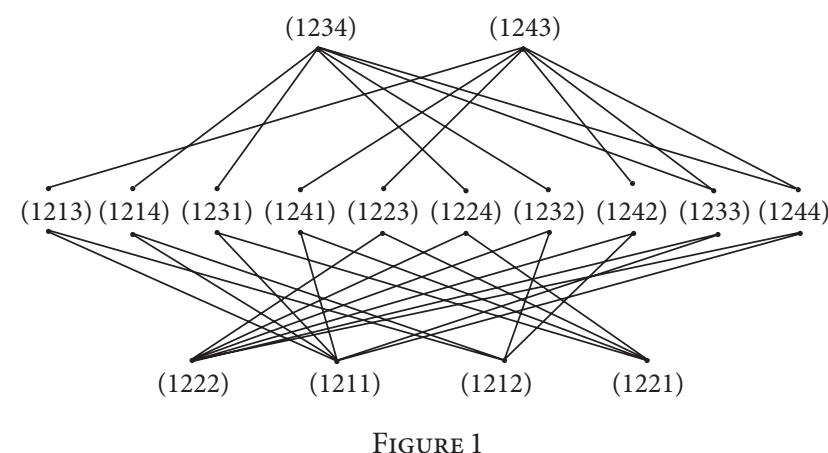

An element $\beta \in \operatorname{Fix}(X, Y)$ is called an upper cover for $\alpha \epsilon$ $\operatorname{Fix}(X, Y)$ if $\alpha<\beta$ and there is no $\gamma \in \operatorname{Fix}(X, Y)$ such that $\alpha<\gamma<\beta$; lower covers are defined dually.

Lemma 10. Assume that $\emptyset \neq Y \subsetneq X$ and let $\alpha \in \operatorname{Fix}(X, Y)$. Then the following statements hold.

(1) If $\alpha$ is not minimal in $\operatorname{Fix}(X, Y)$, then there is some lower cover of $\alpha$ in $\operatorname{Fix}(X, Y)$.

(2) If $\alpha$ is not maximal in $\operatorname{Fix}(X, Y)$, then there is some upper cover of $\alpha$ in $\operatorname{Fix}(X, Y)$.

Proof. (1) Let $\alpha \in \operatorname{Fix}(X, Y)$ be not minimal. By Theorem 8, $\alpha$ is not a minimal idempotent. So we can write

$$
\alpha=\left(\begin{array}{ll}
A_{i} & B_{j} \\
y_{i} & b_{j}
\end{array}\right),
$$

where $J \neq \emptyset$. Define $\beta$ as in the proof of Theorem 8 , we get $\beta<\alpha$. Suppose that there is $\lambda \in \operatorname{Fix}(X, Y)$ such that $\beta \leq \lambda \leq$ $\alpha$. Then by Theorem $1, X \beta \subseteq X \lambda \subseteq X \alpha$ and thus $X \beta \backslash Y \subseteq$ $X \lambda \backslash Y \subseteq X \alpha \backslash Y$. Since $X \alpha \backslash Y=(X \beta \backslash Y) \cup\left\{b_{j_{0}}\right\}$ which implies $X \lambda \backslash Y=X \beta \backslash Y$ or $X \lambda \backslash Y=X \alpha \backslash Y$, thus $\lambda=\beta$ or $\lambda=\alpha$ by Corollary 3. Therefore, $\beta$ is a lower cover of $\alpha$.

(2) The proof is similar to (1), using $\beta$ or $\gamma$ from the proof of Theorem 9 as appropriate.

Now, we aim to find the number of upper covers of minimal elements and the number of lower covers of maximal elements when $X$ is a finite set. The following lemma is needed in finding such numbers.

Lemma 11. Assume that $\emptyset \neq Y \subsetneq X$ and let $\alpha, \beta \in \operatorname{Fix}(X, Y)$ with $\alpha<\beta$. Then $\beta$ is an upper cover of $\alpha$ if and only if $|X \beta|$ $X \alpha \mid=1$.

Proof. Write

$$
\alpha=\left(\begin{array}{ll}
A_{i} & B_{j} \\
y_{i} & b_{j}
\end{array}\right)
$$

Since $\alpha<\beta$, we can write

$$
\beta=\left(\begin{array}{lll}
A_{i}^{\prime} & C_{j} & C_{k} \\
y_{i} & b_{j} & b_{k}
\end{array}\right),
$$


where $y_{i} \in A_{i}^{\prime} \subseteq A_{i}, C_{j} \subseteq B_{j}$, and $C_{k}$ is contained in either $A_{i}$ for some $i$ or $B_{j}$ for some $j$. We get $|K|=|X \beta \backslash X \alpha|$.

Assume that $\beta$ is an upper cover of $\alpha$. If $|X \beta \backslash X \alpha|=0$, then $X \beta=X \alpha$ which implies that $\beta=\alpha$, a contradiction. For the case $|X \beta \backslash X \alpha|>1$, we choose $k_{0} \in K$ and hence $C_{k_{0}} \subseteq A_{i_{0}}$ for some $i_{0} \in I$ or $C_{k_{0}} \subseteq B_{j_{0}}$ for some $j_{0} \in J$. Assume that $C_{k_{0}} \subseteq A_{i_{0}}$ (the other case being similar). Let $I^{\prime}=I \backslash\left\{i_{0}\right\}$ and $K^{\prime}=K \backslash\left\{k_{0}\right\}$. Define

$$
\gamma=\left(\begin{array}{cccc}
A_{i_{0}}^{\prime} \cup C_{k_{0}} & A_{i^{\prime}}^{\prime} & C_{j} & C_{k^{\prime}} \\
y_{i_{0}} & y_{i^{\prime}} & b_{j} & b_{k^{\prime}}
\end{array}\right) \in \operatorname{Fix}(X, Y) .
$$

Since $K^{\prime} \neq \emptyset$, we get $\alpha<\gamma<\beta$, a contradiction. Therefore, $|X \beta \backslash X \alpha|=1$. (1).

The converse is proved in similar fashion to Lemma 10

Let $X$ be a finite set with $n$ elements and $Y$ a nonempty proper subset of $X$ with $r$ elements. If $|Y|=1$, then $\operatorname{Fix}(X, Y)$ has unique minimal element, say $\alpha=\left(\begin{array}{l}X \\ y\end{array}\right)$. By Lemma 11, each of upper covers of $\alpha$ is of the form $\left(\begin{array}{cc}X \backslash B & B \\ y & b\end{array}\right)$, where $\emptyset \neq$ $B \subseteq X \backslash\{y\}$ and $b \in X \backslash Y$. Since there are $\left(2^{n-1}-1\right)$ ways to choose $B$ and $n-1$ choice of $b$, in this case there are in total $\left(2^{n-1}-1\right)(n-1)$ upper covers of $\alpha$.

If $|X \backslash Y|=1$, then $\operatorname{Fix}(X, Y)$ has unique maximal element, the identity map. Let $I=\{1,2, \ldots, n-1\}, Y=\left\{y_{i}: i \in I\right\}$, and $X \backslash Y=\{b\}$. Then each of lower covers of $1_{X}$ is of the form $\left(\begin{array}{cc}\left\{y_{i_{0}}, b\right\} & y_{i^{\prime}} \\ y_{i_{0}} & y_{i^{\prime}}\end{array}\right)$, where $I^{\prime}=I \backslash\left\{i_{0}\right\}$. Since $i_{0}$ can be chosen from $I$, there are in total $n-1$ lower covers of $1_{X}$.

Theorem 12. Assume that $\emptyset \neq Y \subsetneq X$ and let $\alpha \in \operatorname{Fix}(X, Y)$. Then the following statements hold.

(1) If $\alpha=\left(\begin{array}{c}A_{i} \\ y_{i}\end{array}\right)$ is minimal, then there are

$$
\sum_{i=1}^{r}\left(2^{\left|A_{i}\right|-1}-1\right)(n-r)
$$

upper covers of $\alpha$.

(2) If $\alpha$ is maximal, then there are $(n-r)(n-1)$ lower covers of $\alpha$.

Proof. Since $Y$ is a finite set with $r$ elements, $Y=\left\{y_{1}, \ldots, y_{r}\right\}$ and $I=\{1, \ldots, r\}$.

(1) Let $\alpha=\left(\begin{array}{c}A_{i} \\ y_{i}\end{array}\right)$ be minimal in $\operatorname{Fix}(X, Y)$ and $\beta$ an upper cover of $\alpha$. Then $|X \beta \backslash X \alpha|=1$ by Lemma 11; that is, $X \beta=$ $Y \cup\{b\}$ for some $b \in X \backslash Y$. Since $\pi_{\beta}$ must refine $\pi_{\alpha}$, we can write

$$
\beta=\left(\begin{array}{ll}
A_{i}^{\prime} & B \\
y_{i} & b
\end{array}\right)
$$

where $A_{i}^{\prime} \subseteq A_{i}$ and $\emptyset \neq B \subseteq A_{i_{0}} \backslash\left\{y_{i_{0}}\right\}$ for some $i_{0} \in I$. We claim that $A_{i}^{\prime}=A_{i}$ for all $i \in I \backslash\left\{i_{0}\right\}$. Assume by contrary that there is $i_{1} \in I \backslash\left\{i_{0}\right\}$ such that $A_{i_{1}}^{\prime} \subsetneq A_{i_{1}}$. Let $B_{1}=A_{i_{1}} \backslash A_{i_{1}}^{\prime}$.
So $\emptyset \neq B_{1} \cap A_{i}^{\prime} \subseteq A_{i}$ for some $i \neq i_{1}$, but $B_{1} \subseteq A_{i_{1}}$; that is $A_{i} \cap A_{i_{1}} \neq \emptyset$, a contradiction. So we can write

$$
\beta=\left(\begin{array}{cccc}
A_{i^{\prime}} & A_{i_{0}} \backslash B & B \\
y_{i^{\prime}} & y_{i_{0}} & b
\end{array}\right),
$$

where $I^{\prime}=I \backslash\left\{i_{0}\right\}$. Since there are $2^{\left|A_{i_{0}}\right|-1}-1$ ways to choose $B$ and $n-r$ choices of $b$, in this case $\beta$ can have $\left(2^{\left|A_{i_{0}}\right|-1}-1\right)(n-r)$ forms, but $i_{0}$ can be chosen from $I=\{1, \ldots, r\}$, so that there are in total $\sum_{i=1}^{r}\left(2^{\left|A_{i}\right|-1}-1\right)(n-r)$ upper covers of $\alpha$.

(2) Assume that $\alpha$ is maximal. Then $\alpha$ is a bijection and we can write

$$
\alpha=\left(\begin{array}{ll}
y_{i} & b_{j} \\
y_{i} & c_{j}
\end{array}\right)
$$

where $J=\{1, \ldots, n-r\}$ and $\left\{b_{j}: j \in J\right\}=X \backslash Y=\left\{c_{j}\right.$ : $j \in J\}$. Let $\beta$ be a lower cover of $\alpha$. Then $|X \alpha \backslash X \beta|=1$; that is, $X \beta=X \alpha \backslash\left\{c_{j_{0}}\right\}$ for some $j_{0} \in J$. Let $J^{\prime}=J \backslash\left\{j_{0}\right\}$ and $b_{j^{\prime}} \in\left\{b_{j}: j \in J^{\prime}\right\}$. So $b_{j^{\prime}} \alpha=c_{j^{\prime}} \in X \beta \backslash Y$, then $b_{j^{\prime}} \alpha=b_{j^{\prime}} \beta$ since $\beta<\alpha$. Hence $x \alpha=x \beta$ for all $x \in X \backslash\left\{b_{j_{0}}\right\}$ and $b_{j_{0}} \beta=y_{i_{0}}$ for some $i_{0} \in I$ or $b_{j_{0}} \beta=b_{j_{1}} \beta=c_{j_{1}}$ for some $j_{1} \in J^{\prime}$. Thus

$$
\beta=\left(\begin{array}{ccc}
\left\{y_{i_{0}}, b_{j_{0}}\right\} & y_{i^{\prime}} & b_{j^{\prime}} \\
y_{i_{0}} & y_{i^{\prime}} & c_{j^{\prime}}
\end{array}\right),
$$

where $I^{\prime}=I \backslash\left\{i_{0}\right\}$, or

$$
\beta=\left(\begin{array}{ccc}
y_{i} & \left\{b_{j_{1}}, b_{j_{0}}\right\} & b_{k} \\
y_{i} & c_{j_{1}} & c_{k}
\end{array}\right),
$$

where $K=J \backslash\left\{j_{0}, j_{1}\right\}$. For the first form and the second form, the numbers of ways of placing $b_{j_{0}}$ is $r$ and $n-r-1$, respectively. So the total number of ways of placing $b_{j_{0}}$ is $n-1$. But $j_{0}$ varies in the index set $J$; hence there are in total $(n-1)(n-r)$ lower covers of $\alpha$.

\section{Competing Interests}

The authors declare that there is no conflict of interests regarding the publication of this paper.

\section{Acknowledgments}

This research was supported by Chiang Mai University.

\section{References}

[1] R. Hartwig, "How to partially order regular elements," Mathematica Japonica, vol. 35, pp. 1-13, 1980.

[2] K. S. Nambooripad, "The natural partial order on a regular semigroup," Proceedings of the Edinburgh Mathematical Society (Series 2), vol. 23, no. 3, pp. 249-260, 1980.

[3] H. Mitsch, "A natural partial order for semigroups," Proceedings of the American Mathematical Society, vol. 97, no. 3, pp. 384-388, 1986. 
[4] G. Kowol and H. Mitsch, "Naturally ordered transformation semigroups," Monatshefte für Mathematik, vol. 102, no. 2, pp. $115-138,1986$.

[5] C. Namnak and P. Preechasilp, "Natural partial orders on the semigroup of binary relations," Thai Journal of Mathematics, vol. 4, no. 3, pp. 39-50, 2006.

[6] V. H. Fernandes and J. Sanwong, "On the ranks of semigroups of transformations on a finite set with restricted range," Algebra Colloquium, vol. 21, no. 3, pp. 497-510, 2014.

[7] K. Sangkhanan and J. Sanwong, "Partial orders on semigroups of partial transformations with restricted range," Bulletin of the Australian Mathematical Society, vol. 86, no. 1, pp. 100-118, 2012.

[8] P. Honyam and J. Sanwong, "Semigroups of transformations with fixed sets," Quaestiones Mathematicae. Journal of the South African Mathematical Society, vol. 36, no. 1, pp. 79-92, 2013.

[9] Y. Chaiya, P. Honyam, and J. Sanwong, "Maximal subsemigroups and finiteness conditions on transformation semigroups with fixed sets," Turkish Journal of Mathematics, In press.

[10] P. M. Higgins, “The Mitsch order on a semigroup," Semigroup Forum, vol. 49, no. 2, pp. 261-266, 1994. 


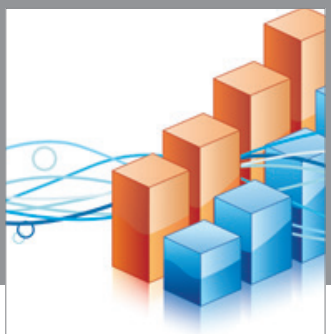

Advances in

Operations Research

vatem alat4

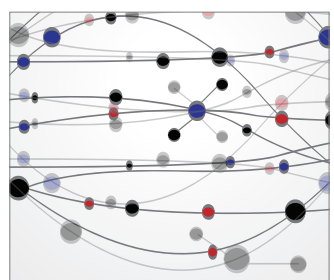

\section{The Scientific} World Journal
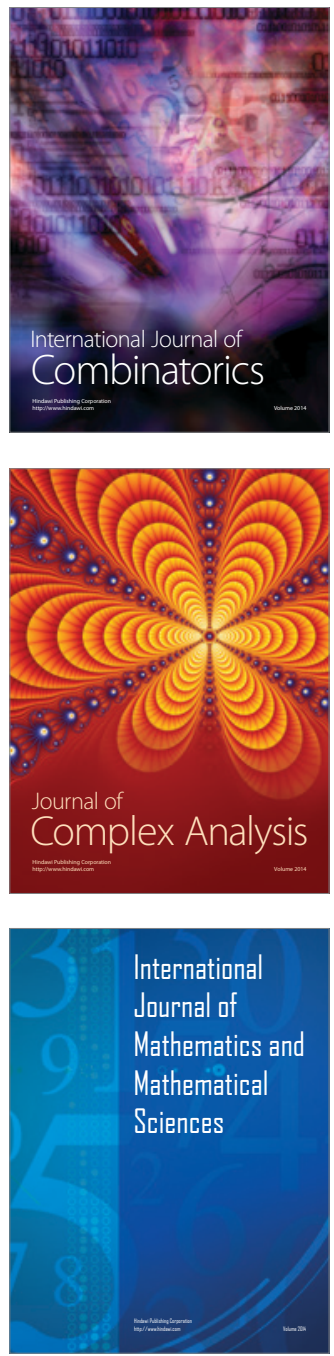
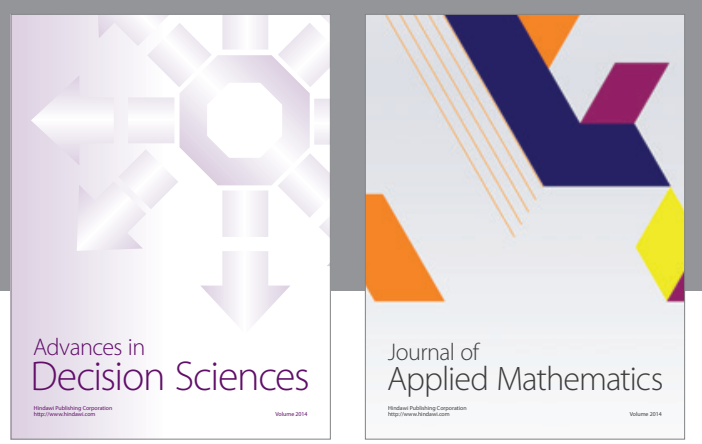

Algebra

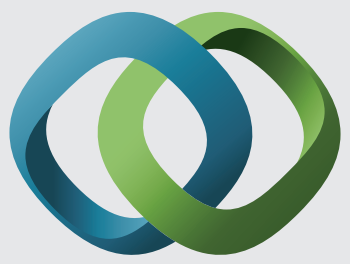

\section{Hindawi}

Submit your manuscripts at

http://www.hindawi.com
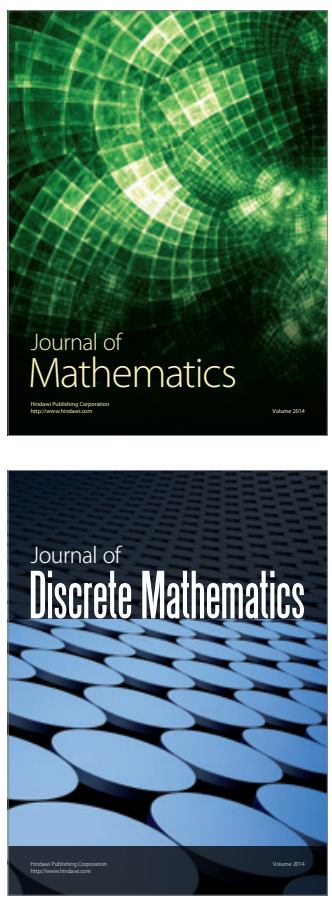

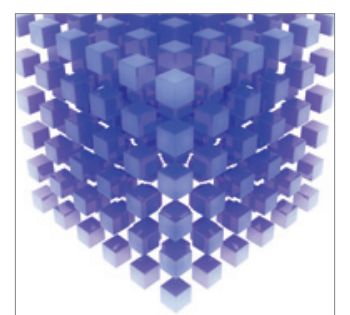

Mathematical Problems in Engineering
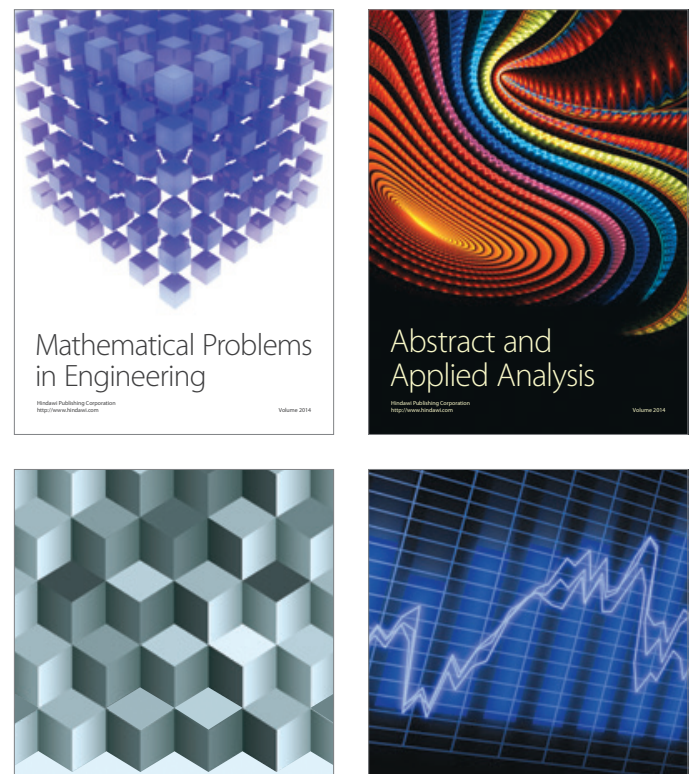

Journal of

Function Spaces

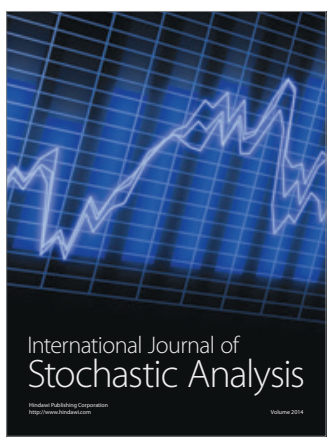

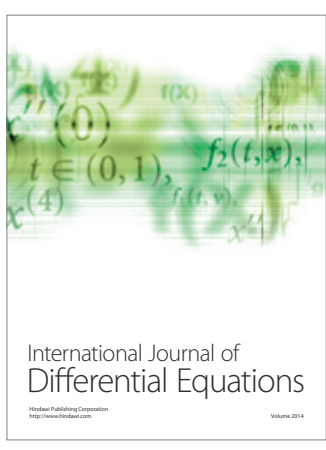
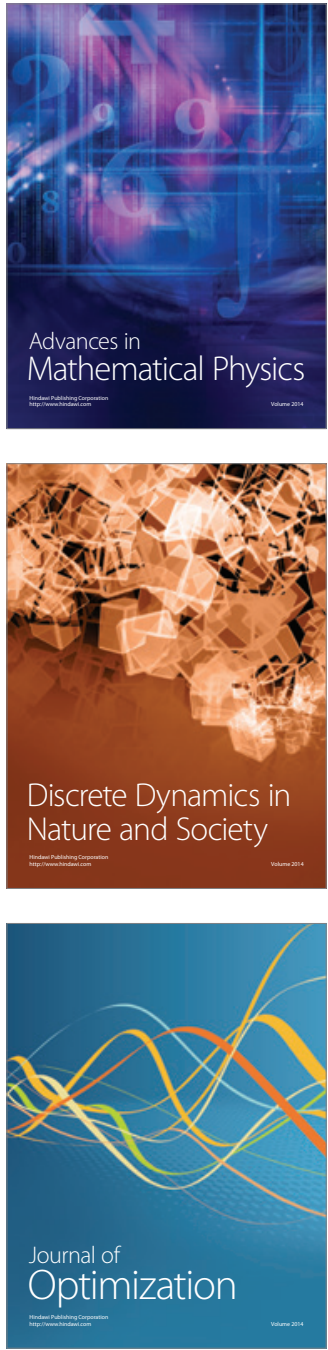